\title{
ON REALIZATION OF SPLITTING OBSTRUCTIONS IN BROWDER-LIVESAY GROUPS FOR CLOSED MANIFOLD PAIRS
}

\author{
P. M. AKHMETIEV ${ }^{1}$, A. CAVICCHIOLI ${ }^{2}$ AND D. REPOVŚ ${ }^{3}$ \\ ${ }^{1}$ IZMIRAN, 142092 Troitsk, Moscow Region, Russia (akhmetev@izmiran.rssi.ru) \\ ${ }^{2}$ Dipartimento di Matematica, Università degli studi di Modena, Via Campi 213/B, \\ 41100 Modena, Italy (albertoc@unimo.it) \\ ${ }^{3}$ Institute of Mathematics, Physics and Mechanics, University of Ljubljana, \\ 1001 Ljubljana, POB 2964, Slovenia (dusan.repovs@fmf.uni-lj.si)
}

(Received 30 April 1997)

\begin{abstract}
We consider some new types of realization problem for obstructions in the Browder-Livesay groups by homotopy equivalences of closed manifold pairs. We give several examples of calculations. We also consider relations with classical surgery problems.
\end{abstract}

Keywords: K-theory; Browder-Livesay groups; splitting obstruction

AMS 1991 Mathematics subject classification: Primary 57R67

Secondary $57 \mathrm{Q}$

\section{Introduction}

One of the central problems of surgery theory for TOP or PL manifolds is the realization problem for obstructions in the Wall groups by normal maps of closed manifolds. This problem has been solved for a large class of manifolds with different fundamental groups (cf. $[6,8])$. We consider a new type of this problem, namely, we study the following question.

Under which conditions can various splitting obstructions be realized by homotopy equivalences of closed manifolds?

In [9] this problem was considered for special cases (cf. Example 2.3). It turns out that it is closely related to the problem of classification of homeomorphisms of closed nonsimply connected manifolds, up to isotopy (cf. Corollaries 2.7 and 2.8). On the other hand, it is also connected with the study of the obstruction groups LS to splitting along a submanifold whose fundamental group maps onto the fundamental group of the manifold itself (cf. Proposition 2.10). 


\section{Main results}

Let $Y$ be a closed connected topological manifold of dimension $n+1 \geqslant 6, X \subset Y$ a submanifold, and $f: M \rightarrow Y$ a simple homotopy equivalence, $[f] \in \mathcal{S}^{\mathrm{TOP}}(Y)$. Denote $N=f^{-1}(X)$. The question is whether there exists a map $f_{1}: M \rightarrow Y$ which is homotopic to $f$ and such that the restrictions

$$
\left.f_{1}\right|_{N}: N \rightarrow X \quad \text { and }\left.\quad f_{1}\right|_{M \backslash N}: M \backslash N \rightarrow Y \backslash X
$$

are simple homotopy equivalences of topological manifolds?

The map $f_{1}: M \rightarrow Y$ is called a splitting of $f$ along $X$ and the question about its existence is called the splitting problem. If $Y$ is a manifold with boundary $\partial Y$, then the splitting problem still makes sense; one must only assume that it is already solved on the boundary $\partial Y$, i.e. that the map $\left.f\right|_{\partial M}: \partial M \rightarrow \partial Y$ has been split along $\partial X \subset \partial Y$, and that the homotopies between $f_{1}$ and $f$ remain fixed on $\partial Y$, where $[f] \in \mathcal{S}^{\mathrm{TOP}}(Y)=$ $[Y / \partial Y, G / \mathrm{TOP}]=H_{n+1}(Y ; \mathbb{L})$.

In the case when $X$ is a one-sided codimension one submanifold, the splitting problem was studied in $[\mathbf{2}, \mathbf{9}]$ for special cases, and in [14] for the general case. Algebraic tools were developed in $[\mathbf{1 1}, \mathbf{1 2}]$. It turned out that in the case when the inclusion-induced homomorphism $i: \pi_{1}(X) \rightarrow \pi_{1}(Y)$ is an isomorphism (we shall hereafter call such manifold pairs $(Y, X)$ admissible $)$, the group of obstructions to the splitting problem is defined algebraically and it depends only on dimension $n(\bmod 4)$, as well as on the homomorphisms of fundamental groups

$$
\ell: \pi=\pi_{1}(Y \backslash X) \rightarrow G=\pi_{1}(Y)
$$

induced by the embedding $Y \backslash X \rightarrow Y$. For an admissible pair, the homomorphism $\ell$ is an embedding of a subgroup of index 2. The groups $\pi$ and $G$ are equipped with the homomorphisms

$$
\omega_{Y \backslash X}: \pi \rightarrow \mathbb{Z}_{2} \quad \text { and } \quad \omega_{Y}: G \rightarrow \mathbb{Z}_{2}
$$

which are defined geometrically as the orientation homomorphisms for the manifolds $Y \backslash X$ and $Y$, respectively.

We shall denote by $G^{-}$the group distinguished from $G$ by the value of $\omega_{Y}$ on $G \backslash \pi$. It is not difficult to check that the homomorphism $\omega_{Y \backslash X}$ is induced by the homomorphism $\omega_{Y}$, under the embedding $\ell$. According to [14], the obstruction group is denoted by $\mathrm{LN}_{n}(\pi \rightarrow$ $G)$. This group is the Witt group of quadratic $(n / 2)$-forms (respectively $((n-1) / 2)$ formations) over the group ring $\mathbb{Z}[\pi]$, equipped with the antistructure (cf. [12]).

In the present paper we consider the calculation in the simplest case $G=\pi \times \mathbb{Z}_{2}$. In this case we have $\operatorname{LN}_{n}(\pi \rightarrow G)=L_{n+1+\omega(t)}(\pi)$, where $t$ is the generator in $\mathbb{Z}_{2}$ and $\omega(t)=+1$ or -1 . In the general case, the obstruction group is defined as the Wall group over the ring with antistructure $\left(\mathbb{Z}[\pi], \alpha, t^{2}\right)$, where $t \in G \backslash \pi, \alpha: \mathbb{Z}[\pi] \rightarrow \mathbb{Z}[\pi]$ is the automorphism given by $\alpha(u)=t u t^{-1}$. The obstruction to splitting of $f$ along $X$ is denoted by $u(f) \in \mathrm{LN}_{n}(\pi \rightarrow G)$. 
We now introduce some key definitions. For an arbitrary admissible pair $(Y, X)$, we shall denote by $\mathrm{BL}(Y, X) \subset \mathrm{LN}_{n}(\pi \rightarrow G)$ the subset consisting of the elements which can be realized as an obstruction, under some choice of the simple homotopy equivalence $f: M \rightarrow Y$. Let us denote by $\widetilde{\mathrm{BL}}(Y, X) \subset \mathrm{BL}(Y, X)$ the subset of those obstructions $u(f)$ which can be realized by a simple homotopy equivalence $f: M \rightarrow Y$ having the trivial normal invariant

$$
S(f) \in[Y ; G / \mathrm{TOP}]=H_{n+1}(Y ; \mathbb{L}) .
$$

The subsets $\widetilde{\mathrm{BL}}(Y, X)$ and $\mathrm{BL}(Y, X)$ can be described by means of the Ranicki assembly construction and they are well-defined abelian groups (see $\S 3$ ).

Proposition 2.1 (C. T. C. Wall). Let $(Y, X)$ be an admissible pair of manifolds with non-empty boundaries $\partial Y \supset \partial X$, such that the pair $(\partial Y, \partial X)$ is itself admissible, and the homomorphisms $\pi_{1}(\partial X) \rightarrow \pi_{1}(X)$ and $\pi_{1}(\partial Y) \rightarrow \pi_{1}(Y)$ are isomorphisms. Then the group $\mathrm{BL}(Y, X)$ is equal to the entire group $\operatorname{LN}_{n}(\pi \rightarrow G)$.

Problem 2.2. Let $(Y, X)$ be an admissible pair of closed manifolds. Describe the subgroups $\widetilde{\mathrm{BL}}(Y, X)$ and $\mathrm{BL}(Y, X)$ of the group $\mathrm{LN}_{n}(\pi \rightarrow G)$.

We remark that analogous problem can be asked for the groups $\mathrm{LP}(Y, X)$ (cf. [10]). Let us calculate the groups $\mathrm{BL}(Y, X)$ and $\widetilde{\mathrm{BL}}(Y, X)$ for some specific examples of manifold pairs.

Example 2.3 (cf. [9]). Let $Y=\mathbb{R} P^{7} \supset \mathbb{R} P^{6}=X, \pi_{1}(Y)=\mathbb{Z}_{2}^{+}$and $\operatorname{dim} X \equiv 2$ $(\bmod 4)$. Then the subgroups $\widetilde{\mathrm{BL}}(Y, X)$ and $\mathrm{BL}(Y, X)$ coincide with the entire group $\mathrm{LN}_{2}\left(0 \rightarrow \mathbb{Z}_{2}^{+}\right)=L_{0}(1)=\mathbb{Z}$.

Example 2.4. Let $Y=\mathbb{R} P^{2} \times \mathbb{R} P^{5} \supset \mathbb{R} P^{2} \times \mathbb{R} P^{4}=X, \pi_{1}(Y)=\mathbb{Z}_{2}^{-} \oplus \mathbb{Z}_{2}^{+}$and $\operatorname{dim} X \equiv 2(\bmod 4)$. Then the subgroup $\widetilde{\mathrm{BL}}(Y, X)$ is trivial, whereas $\mathrm{BL}(Y, X)$ coincides with the entire group $L_{2}\left(\mathbb{Z}_{2}^{-} \rightarrow \mathbb{Z}_{2}^{-} \oplus \mathbb{Z}_{2}^{+}\right)=L_{0}\left(\mathbb{Z}_{2}^{-}\right)=\mathbb{Z}_{2}$.

Example 2.5. Let $Y=\mathbb{R} P^{3} \times \mathbb{R} P^{3} \supset \mathbb{R} P^{2} \times \mathbb{R} P^{3}=X, \pi_{1}(Y)=\mathbb{Z}_{2}^{+} \oplus \mathbb{Z}_{2}^{+}$and $\operatorname{dim} X \equiv 1(\bmod 4)$. Then the subgroups $\widetilde{\mathrm{BL}}(Y, X)$ and $\mathrm{BL}(Y, X)$ agree with the entire group $\mathrm{LN}_{1}\left(\mathbb{Z}_{2}^{+} \rightarrow \mathbb{Z}_{2}^{+} \oplus \mathbb{Z}_{2}^{+}\right)=L_{3}\left(\mathbb{Z}_{2}^{+}\right)=\mathbb{Z}_{2}$.

Example 2.6. Let $Y=\mathbb{R} P^{3} \times \mathbb{R} P^{3} \times \mathbb{R} P^{3} \times \mathbb{R} P^{3} \supset \mathbb{R} P^{3} \times \mathbb{R} P^{3} \times \mathbb{R} P^{3} \times \mathbb{R} P^{2}=X$, $\pi_{1}(Y)=\left(\mathbb{Z}_{2}^{+}\right)^{4}$ and $\operatorname{dim} X \equiv 3(\bmod 4)$. Then the subgroups $\widetilde{\mathrm{BL}}(Y, X)$ and $\mathrm{BL}(Y, X)$ are trivial, $\mathrm{LN}_{3}\left(\left(\mathbb{Z}_{2}^{+}\right)^{3} \rightarrow\left(\mathbb{Z}_{2}^{+}\right)^{4}\right)=L_{1}\left(\left(\mathbb{Z}_{2}^{+}\right)^{3}\right)=\mathbb{Z}_{2}$.

Let us consider an application, connected with the group $\widetilde{\mathrm{BL}}(Y, X)$. Let $f, g: M \rightarrow M$ be any homeomorphisms and let $\operatorname{dim} M \geqslant 5$. We shall say that $f$ and $g$ are connected via a pseudoisotopy or concordant if there exists a homeomorphism $F: M \times[0,1] \rightarrow M \times[0,1]$ such that $\left.F\right|_{M \times\{0\}}=f$ and $\left.F\right|_{M \times\{1\}}=g$.

Proposition 2.7. There exists an infinite set of homeomorphisms

$$
f_{i}: \mathbb{R} P^{3} \times S^{3} \rightarrow \mathbb{R} P^{3} \times S^{3},
$$

homotopic to the identity map, such that no two among them are concordant. 
Proposition 2.8. There exists a homeomorphism

$$
f: \mathbb{R} P^{3} \times \mathbb{R} P^{3} \times S^{3} \rightarrow \mathbb{R} P^{3} \times \mathbb{R} P^{3} \times S^{3},
$$

which is homotopic, but not concordant to the identity homeomorphism.

The main result of this paper-Theorem 2.10 - is related to the following problem.

Problem 2.9. Does the subgroup $\mathrm{BL}(Y, X)$ depend on the homotopy type of the manifold $Y$ ?

Theorem 2.10. Suppose that the element $a \in \mathrm{BL}(Y, X) \subset \mathrm{LN}_{n}(\pi \rightarrow G)$ can be realized as the obstruction $a=u(f)$ to the splitting, for some choice of $f$ and for an arbitrary admissible pair $(Y, X)$ of closed manifolds with the given $\pi_{1}(Y)=G$ and $n=\operatorname{dim} X$. Then the surgery obstruction $b \in L_{n}\left(G^{-}\right)$of the normal map $f: N \rightarrow X$ lies in the image of the homomorphism $L_{n}\left(\underset{i=1}{*} \mathbb{Z}^{ \pm}\right) \rightarrow L_{n}\left(G^{-}\right)$, induced by some $\underset{k}{\operatorname{copimorphism}}$ $\underset{i=1}{*} \mathbb{Z}^{ \pm} \rightarrow G^{-}$, which preserves the orientation of $\omega$. (Each element in $L_{n}\left(\underset{i=1}{*} \mathbb{Z}^{ \pm}\right.$) lies in the image of $L_{n}\left(\mathbb{Z}^{ \pm}\right)$, for some $\operatorname{map} \mathbb{Z}^{ \pm} \rightarrow \underset{i=1}{*} \mathbb{Z}^{ \pm}$(cf. [4]).) In particular, $\alpha$ is not the Arf invariant in codimension $\geqslant 3$ (cf. [5]).

The subgroup $\widetilde{\mathrm{BL}}(Y, X) \subset \mathrm{BL}(Y, X)$ is defined algebraically (see the next section) and, therefore, this subgroup does not depend on the admissible pair $(Y, X)$. Examples 2.3 and 2.5 show that the group $\widetilde{\mathrm{BL}}(Y, X)$ (and therefore $\mathrm{BL}(Y, X)$ ) is non-trivial for some pair $(\pi \subset G, n)$. We also note that any non-trivial element of $\widetilde{\mathrm{BL}}(Y, X)$ yields an example of a non-zero element of $\mathrm{LN}_{n}(\pi \rightarrow G)$ which can be realized as the splitting obstruction, for an arbitrary manifold pair $(Y, X)$, with given $\pi_{1}(Y), n=\operatorname{dim} X$.

The proofs are organized in the following way. In $\S 3$ we recall algebraic properties of the splitting obstruction group for admissible pairs of manifolds and we present the computations of Examples 2.3-2.6. In $\S 4$ we recall some algebraic properties of the splitting obstruction group when the pair $(Y, X)$ is inadmissible and the inclusion $X \subset Y$ induces an epimorphism $\pi_{1}(X) \rightarrow \pi_{1}(Y)$. At the end of the section we prove the main theorem.

\section{Admissible manifold pairs}

Proof of Proposition 2.1. Let $(Y, \partial Y) \supset(X, \partial X)$ be an admissible pair. Consider a neighbourhood $U \supset \partial Y$ of the boundary $\partial Y$ such that $U=\partial Y \times I$, where $I=[0,1]$. Assume that $\partial Y \times\{1\}$ is identified with the boundary of the manifold $Y$.

According to a result of $[\mathbf{1 4}]$, there exists a simple homotopy equivalence

$$
F:\left(W, W_{0} \cup W_{1}\right) \rightarrow(Y \times I, \partial Y \times\{0\} \cup \partial Y \times\{1\})
$$

such that $\left.F\right|_{W_{0}}$ is a homeomorphism and $\left.F\right|_{W_{0}}$ is a simple homotopy equivalence, split along the submanifold $\partial X \times\{1\} \subset \partial Y \times\{1\}$, where the obstruction to splitting of $F$ 
along the submanifold $\partial X \times I \subset \partial Y \times I$ (rel $\partial$ ) coincides with an arbitrarily chosen obstruction $a$ from the group $\operatorname{LN}_{n}(\pi \rightarrow G)$. We now glue the map $F$ with the identity map Id : $Y \backslash U \rightarrow Y \backslash U$, along the boundary $\partial Y, f=F \cup_{\partial Y}$ Id. Then $a$ is the obstruction to splitting of $f$ along $X$.

We recall some properties of the splitting obstruction group. Consider the following diagram [11, p. 582]:

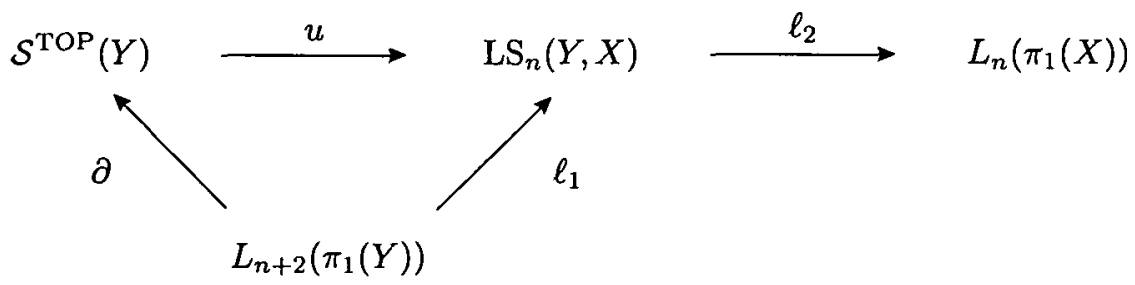

The map $u$ gives the splitting obstruction of a simple homotopy equivalence $f: M \rightarrow Y$ along $X \subset Y,[f] \in \mathcal{S}^{\mathrm{TOP}}(Y)=[Y / \partial Y, G / \mathrm{TOP}]=H_{n+1}(Y, \mathbb{L})$. The map $\partial$ is a map in the algebraic surgery exact sequence $[12, \mathrm{p} .8], \ell_{1}=u \circ \partial$. The map $\ell_{2}$ gives the surgery obstruction of the restriction $\left.f\right|_{N}: N \rightarrow X$.

By construction we have that $\widetilde{\mathrm{BL}}(Y, X)=\operatorname{Im} \ell_{1}$ and $\operatorname{BL}(Y, X)=\operatorname{Im} u$. The set $\mathcal{S}^{\mathrm{TOP}}(Y)$ is equipped by an abelian group structure, hence the sets $\mathrm{BL}(Y, X)$ and $\widetilde{\mathrm{BL}}(Y, X)$ are abelian groups. If the pair $(Y, X)$ is admissible, we have

(i) $\operatorname{LS}_{n}(Y, X)=\mathrm{LN}_{n-1}(\pi \rightarrow G)$;

(ii) $\ell_{2}=t \circ i: \operatorname{LN}_{n}(\pi \rightarrow G) \rightarrow L_{n}\left(G^{-}\right)$; and

(iii) $\ell_{1}=i$ ! $\circ t^{-1}: L_{n+2}(G) \rightarrow \operatorname{LN}_{n}(\pi \rightarrow G)$;

where $i^{!} \circ t^{-1}$ is a composition of the transfer homomorphism with the scaling homomorphism, $i$ is induced by inclusion $\pi \rightarrow G$.

Recall the usual diagram

$$
\begin{gathered}
L_{n+2}(G ; \omega \circ \psi) \\
\downarrow \\
\cdots L_{n+1}(G, \omega) \rightarrow L_{n+2}(\pi \rightarrow G ; \omega \circ \psi) \rightarrow \operatorname{LN}_{n}(\pi \rightarrow G ; \omega) \rightarrow L_{n}(G ; \omega) \cdots \\
\downarrow \\
L_{n+1}(\pi ; \omega \circ \psi)
\end{gathered}
$$

where $\pi$ is the kernel of the epimorphism $\psi: G \rightarrow \mathbb{Z}_{2}$. Due to the lack of space, we shall only make some remarks about $i$ and $t$. In particular, $\operatorname{LN}_{n}(\pi \rightarrow G ; \omega)$ is isomorphic to 
$L_{n}(\pi$, anti), where anti means the $L$ group with some antistructure: $i$ denotes the inclusion and transfer from $L_{n}\left(\pi\right.$, anti) to $L_{n}\left(G\right.$,anti) and $i^{!}$denotes the transfer going in the opposite direction. The maps $t$ used above are isomorphisms $t: L_{n}\left(G\right.$; anti) $\rightarrow L_{n}(G ; \omega)$ and $t: L_{n}(G ;$ anti $) \rightarrow L_{n-2}(G ; \omega \circ \phi)$.

The final remark needed to understand most of our proofs is that if there exists an element $t \in G \backslash \pi$ such that $t^{2}=e$ (as in the case of all our examples) $\operatorname{LN}_{n}(\pi \rightarrow G ; \omega)=$ $L_{n-2}(\pi ; \omega)$ if $\omega(t)=-1$ or $\operatorname{LN}_{n}(\pi \rightarrow G ; \omega)=L_{n}(\pi ; \omega)$ if $\omega(t)=1$.

We also observe that the map $i^{!} \circ t^{-1}: L_{n+2}(G) \rightarrow \operatorname{LN}_{n}(\pi \rightarrow G)$ in the exact sequence

$$
\cdots \rightarrow L_{n+2}\left(i^{!}: G \rightarrow \pi\right) \rightarrow L_{n+2}(\pi) \rightarrow L_{n}(\pi \rightarrow G) \rightarrow \cdots
$$

is described in $[\mathbf{1 2}$, p. 264] in terms of algebraic Poincaré complexes, whereas the map $t \circ i: \operatorname{LN}_{n}(\pi \rightarrow G) \rightarrow L_{n}\left(G^{-}\right)$in the exact sequence

$$
\cdots \rightarrow L_{n+1}\left(G^{-}\right) \rightarrow L_{n+2}(\pi \rightarrow G) \rightarrow \mathrm{LN}_{n}(\pi \rightarrow G) \stackrel{t \circ i}{\longrightarrow} L_{n}\left(G^{-}\right) \rightarrow \cdots
$$

is described geometrically in [14, Theorem 11.6].

Calculations of Examples 2.3 and 2.5. It suffices to prove that the maps

$$
i^{!}: L_{0}\left(\mathbb{Z}_{2}^{+}\right) \rightarrow L_{0}(1)
$$

and

$$
i^{!}: L_{3}\left(\mathbb{Z}_{2}^{+} \oplus \mathbb{Z}_{2}^{+}\right) \rightarrow L_{3}\left(\mathbb{Z}_{2}\right)
$$

are epimorphisms. This was done in [14].

Calculations of Example 2.4. The map

$$
i^{!}: L_{2}\left(\mathbb{Z}_{2}^{+} \oplus \mathbb{Z}_{2}^{-}\right) \rightarrow L_{2}\left(\mathbb{Z}_{2}^{-}\right)
$$

is trivial. Therefore $\widetilde{\mathrm{BL}}(Y, X)=0$. Next, let $[a] \in L_{2}\left(\mathbb{Z}_{2}^{-}\right) \cong \mathbb{Z}_{2}$ be a generator and consider the quadratic form $b=\left(t \circ i_{*}\right)(a) \in L_{2}\left(\mathbb{Z}_{2}^{-} \oplus \mathbb{Z}_{2}^{-}\right)$. This form corresponds to the obstruction to surgery of the normal map $f^{\prime}: N \rightarrow \mathbb{R} P^{2} \times \mathbb{R} P^{4}$ to a homotopy equivalence.

Let

$$
\left[f^{\prime}\right] \in H_{6}\left(\mathbb{R} P^{2} \times \mathbb{R} P^{4} ; \mathbb{L}(\mathbb{Z})\right)=\sum_{k \in \mathbb{Z}} H_{6-k}\left(\mathbb{R} P^{2} \times \mathbb{R} P^{4} ; L_{k}(\mathbb{Z})\right), \quad A\left(\left[f^{\prime}\right]\right)=[b],
$$

where $A$ is the assembly map in the surgery exact sequence. The form $i^{!}(b)$ over $\mathbb{Z}[\pi]$ is equipped with the Lagrangian $\Lambda \subset i^{!}(b)$ (the trivialization) such that $i^{!}(b)=\Lambda \oplus T \Lambda$, where $T: i^{\prime}(b) \rightarrow i^{\prime}(b)$ is a homomorphism, given by the multiplication of $t \in G \backslash \pi$ (cf. [7]).

The map $\hat{f}^{\prime}: \hat{N} \rightarrow \mathbb{R} P^{2} \times S^{4}$, being a 2-fold covering of $f$, has the trivial normal invariant $[\hat{f}] \in H_{6}\left(\mathbb{R} P^{2} \times S^{4} ; \mathbb{L}_{0}(\mathbb{Z})\right)$ and consequently, the map $f$ can be surgered to a 
homeomorphism $h^{\prime \prime}: N^{\prime \prime} \rightarrow \mathbb{R} P^{2} \times S^{4}$, by means of a normal cobordism $F$, which in turn is determined by the choice of the trivialization $\Lambda^{\prime \prime}$ of the form $A^{!}(f)$.

The trivialization $\Lambda^{\prime \prime}$ need not coincide with the prescribed trivialization $\Lambda$ by which the surgery obstruction $a$ was defined. The homotopy equivalence $h^{\prime}: N^{\prime} \rightarrow \mathbb{R} P^{2} \times S^{4}$, obtained by surgery of $\hat{f}^{\prime}$ in accordance with the trivialization $\Lambda$, differs from the homeomorphism $h^{\prime \prime}$ by the action of some element of the group $L_{3}\left(\mathbb{Z}_{2}^{-}\right)$on the set $\mathcal{S}^{\mathrm{TOP}}\left(\mathbb{R} P^{2} \times S^{4}\right)$ of the homotopy structure on the manifold $\mathbb{R} P^{2} \times S^{4}$. On the other hand, $L_{3}\left(\mathbb{Z}_{2}^{-}\right)=0$ and the map $h^{\prime \prime}$ is homotopic to $h^{\prime}$. Consequently, $h^{\prime}$ is a homeomorphism.

We define $f: M \rightarrow \mathbb{R} P^{2} \times \mathbb{R} P^{5}$ by gluing the equivariant homotopy equivalence

$$
G=F \bigcup_{\mathbb{R} P^{2} \times S^{4}} \rightarrow \mathbb{R} P^{2} \times S^{4} \times[-1,1]
$$

along the boundary $N^{\prime} \cup T N^{\prime}=\partial(G)$ by two standard copies of $\left(\mathbb{R} P^{2} \times \mathbb{D}^{5}, \partial\right)$. The element $a$ is realized as the obstruction to splitting of $f$ along $X$.

Calculation for Example 2.6. The map

$$
t \circ i: L_{1}\left(\left(\mathbb{Z}_{2}^{+}\right)^{3}\right) \rightarrow L_{1}\left(\left(\mathbb{Z}_{2}^{+}\right)^{3} \oplus \mathbb{Z}_{2}^{-}\right)
$$

is an isomorphism. By a theorem of Kharshiladze [8], the single non-trivial element of the group $L_{1}\left(\left(\mathbb{Z}_{2}^{+}\right)^{3} \oplus \mathbb{Z}_{2}^{-}\right)$cannot be realized by a normal map of closed manifolds. Therefore, the non-trivial element of the group $\mathrm{LN}_{3}\left(\left(\mathbb{Z}_{2}^{+}\right)^{3} \rightarrow\left(\mathbb{Z}_{2}^{+}\right)^{4}\right)$ cannot be realized as the splitting obstruction.

Proof of Proposition 2.7. Let $Y=\mathbb{R} P^{3} \times S^{3} \times[0,1]$ and consider the set $\mathcal{S}^{\mathrm{TOP}}(Y)$ of homotopy structures of manifolds $Y, \operatorname{rel} \partial Y$. Then $\mathcal{S}^{\mathrm{TOP}}(Y)$ can be represented by a homotopy equivalence $f: Y \rightarrow Y$, such that the restriction $\left.f\right|_{\partial Y}$ is a homeomorphism.

The group $L_{0}\left(\mathbb{Z}_{2}^{+}\right)$acts on the elements of the set $\mathcal{S}^{\mathrm{TOP}}(Y)$ by means of the map $\eta: L_{0}\left(\mathbb{Z}_{2}^{+}\right) \rightarrow \mathcal{S}^{\mathrm{TOP}}(Y)$ from the surgery exact sequence in the category of manifolds with boundary $\left[12\right.$, p. 208]. Define $f_{i}$ as the result of the action of the element $b_{i} \in L_{0}\left(\mathbb{Z}_{2}^{+}\right)$, where we choose the sequence of elements $b_{i}$ in every residue class with respect to the kernel of the epimorphism.

There is some arbitrariness in the choice of the parametrization of the preimage of the map $f_{i}$, homeomorphic to $Y$. We shall assume that $\left.f_{i}\right|_{\mathbb{R} P^{3} \times S^{3} \times\{0\}}$ is the identity homeomorphism. Then $\left.f_{i}\right|_{3 P^{3} \times S^{3} \times\{1\}}$ is the initial homeomorphism. Denote this homeomorphism by $h_{i}: \mathbb{R} P^{3} \times S^{3} \rightarrow \mathbb{R} P^{3} \times S^{3}$ and let us prove that $h_{i}$ is not concordant to the identity homeomorphism Id : $\mathbb{R} P^{3} \times S^{3} \rightarrow \mathbb{R} P^{3} \times S^{3}$.

Suppose to the contrary. Let

$$
\left.g_{i}\right|_{3 P^{3} \times S^{3} \times\{1\}}=h_{i} \text { and }\left.g_{i}\right|_{3 P^{3} \times S^{3} \times\{2\}}=\mathrm{Id} .
$$

Consider the map

$$
k_{i}=f_{i} \cup g_{i}: \mathbb{R} P^{3} \times S^{3} \times[0,1] \cup_{\mathbb{B} P^{3} \times S^{3} \times\{1\}} \mathbb{R} P^{3} \times S^{3} \times[1,2] \rightarrow \mathbb{R} P^{3} \times S^{3} \times[0,2] .
$$


By construction, $k_{i}$ induces the identity map of the boundary $\mathbb{R} P^{3} \times S^{3} \times\{0,2\}$.

We shall prove that the map $k_{i}$ splits along $\mathbb{R} P^{3} \times S^{3} \times[0,2]$ (rel $\partial$ ). Let $p t \in \mathbb{R} P^{3}$ be the distinguished point and $\alpha \in \pi_{1}\left(\mathbb{R} P^{3} \times S^{3}\right)$ a loop defined as the projection of the path $k_{i}(p t \times[0,2]) \subset \mathbb{R} P^{3} \times S^{3} \times[0,2]$ onto $\mathbb{R} P^{3} \times S^{3}$.

If $\alpha \neq 0$, take an isotopy

$$
\mathcal{I}:[2,3] \times \mathbb{R} P^{3} \times S^{3} \rightarrow[2,3] \times \mathbb{R} P^{3} \times S^{3}
$$

such that $\mathcal{I}([2,3])$ is projected onto $\mathbb{R} P^{3} \times S^{3}$ as a generator in $\pi_{1}\left(\mathbb{R} P^{3} \times S^{3}\right)$. Consider the map

$$
k_{i}^{\prime}=k_{i} \bigcup \mathcal{I}:[0,3] \times \mathbb{R} P^{3} \times S^{3} \rightarrow[0,3] \times \mathbb{R} P^{3} \times S^{3} .
$$

$\{2\} \times \mathbb{R} P^{3} \times S^{3}$

After the reparametrization $[0,3] \rightarrow[0,2]$ we have the map $k_{i}^{\prime \prime}$, under the condition $\alpha=0$. Obviously, the reduced map $k_{i}^{\prime \prime}$ splits (rel $\partial$ ) if and only if the map $k_{i}$ does. We denote below the map $k_{i}^{\prime \prime}$ by $k_{i}$.

We construct the map

$$
p_{i}: \mathbb{R} P^{3} \times S^{3} \times S^{1} \rightarrow \mathbb{R} P^{3} \times S^{3} \times S^{1}
$$

by the identification of the two components of the boundary of the map $k_{i}$. By construction, $p_{i}$ joins via homotopy with the identity map. Indeed, the homomorphisms

$$
\left(p_{i}\right)_{\#}: \pi_{i}\left(\mathbb{R} P^{3} \times S^{3} \times S^{1}\right) \rightarrow \pi_{i}\left(\mathbb{R} P^{3} \times S^{3} \times S^{1}\right)
$$

are identity.

Therefore, the splitting obstruction of the map $p_{i}$ along $\mathbb{R} P^{2} \times S^{3} \times S^{1}$ is zero. The splitting obstruction of the map $k_{i}$ (rel $\partial$ ) along $\mathbb{R} P^{3} \times S^{3} \times I$ can be expressed using the splitting obstruction of the map $p_{i}$ along $\mathbb{R} P^{2} \times S^{3} \times S^{1}$ by the Shaneson decomposition of the Wall group

$$
L^{s}\left(\pi \times \mathbb{Z}^{+}\right)=L_{n-1}^{n}(\pi) \oplus L_{n}^{s}(\pi),
$$

see [13]. Therefore $k_{i}$ splits over $\mathbb{R} P^{2} \times S^{3} \times I$ (rel $\left.\partial\right)$.

On the other hand, the obstruction $\sigma\left(k_{i}\right)$ to the splitting of $k_{i}$ along the submanifold

$$
\mathbb{R} P^{2} \times S^{3} \times[0,2] \subset \mathbb{R} P^{3} \times S^{3} \times[0,2]
$$

is non-zero. Moreover, $\sigma\left(k_{i}\right) \neq \sigma\left(k_{j}\right)$ for any $i \neq j$ (see the calculation of Example 2.3). Therefore the homeomorphisms $k_{i}$ from the group Homeo $\left(\mathbb{R} P^{3} \times S^{3}\right)$ belong to different residue classes with respect to the subgroup induced by the relation of concordance.

Proof of Proposition 2.8. The argument is analogous to the proof of Corollary 2.7: apply results from the calculations of Example 2.5. 


\section{Inadmissible manifold pairs}

We recall basic properties of the splitting obstruction group $\operatorname{LS}_{n}(Y, X)$ if the pair $(Y, X)$ is inadmissible and the homomorphism $\pi_{1}(X) \rightarrow \pi_{1}(Y)$ is an epimorphism. The group includes into the exact sequence (see [1])

$$
\cdots L_{n+1}\left(\pi_{1}(X) \rightarrow \pi_{1}(Y)\right) \rightarrow \operatorname{LS}_{n}(Y, X) \stackrel{p}{\rightarrow} \operatorname{LN}_{n}(\pi \rightarrow G) \stackrel{d}{\rightarrow} L_{n}\left(\pi_{1}(X) \rightarrow \pi_{1}(Y)\right) \rightarrow \cdots
$$

Let $(Y, X)$ be an admissible pair, $X^{\prime} \subset Y \times\{1\}$ a manifold, concordant to $X \times\{0\}$ in $Y \times[0,1]$, and $\pi_{1}\left(X^{\prime}\right) \rightarrow \pi_{1}(Y)$ an epimorphism. For an arbitrary $[f] \in \mathcal{S}^{\mathrm{TOP}}(Y)$ the splitting obstruction $u(f)$ lies in the subgroup $\operatorname{Im}(p)=\operatorname{Ker}(d)$ in the sequence (4.1). Some examples give $\operatorname{Ker}(d) \neq \operatorname{LN}_{n}(Y, X)$ (cf. [1, Diagram 7]).

Example 4.1 (A. F. Kharshiladze). Let $Y=L^{3}\left(\mathbb{Z}_{4}\right) \times S^{N}$, where $L^{3}\left(\mathbb{Z}_{4}\right)=S^{3} / \mathbb{Z}_{4}$ is the standard lens $\mathbb{Z}_{4}$-space. There exists a one-sided submanifold $K^{2} \times S^{N} \subset Y$, where $K^{2}$ is the Klein bottle. We have

(i) $\pi_{1}\left(K^{2}\right)=\underset{2 \mathbb{Z}}{\mathbb{Z}} \mathbb{Z}=\left\{t_{1}, t_{2} \mid t_{1}^{2}=t_{2}^{2}\right\}$;

(ii) $\pi_{1}\left(L^{3}\left(\mathbb{Z}_{4}\right)\right)=\mathbb{Z}_{4}=\left\{t \mid t^{4}=1\right\}$;

(iii) $\underset{2 \mathbb{Z}}{\mathbb{Z}} \underset{2 \mathbb{Z}}{*} \mathbb{Z}_{4}$

(iv) $i_{\#}\left(t_{1}\right)=i_{\#}\left(t_{2}\right)=t$; and

(v) $\omega_{K^{2}}\left(t_{1}\right)=\omega_{K^{2}}\left(t_{2}\right)=-1$.

Therefore for an admissible pair $(Y, X)$ the splitting obstruction lies in:

$$
u(f) \in \operatorname{Ker}\left[\mathrm{LN}_{n}(Y, X) \stackrel{d}{\rightarrow} L_{n}\left(\underset{2 \mathbb{Z}}{*} \underset{\mathbb{Z}}{\mathbb{Z}} \rightarrow \mathbb{Z}_{4}^{-}\right)\right] .
$$

Definition 4.2. Let $(Y, X)$ be an admissible pair of closed manifolds. We shall say that the manifold $Y$ is exceptional if there exists another one-sided submanifold $X^{\prime} \subset Y$ in the same concordance class, such that $X^{\prime}$ has a free (non-abelian) fundamental group and the homomorphism $\pi_{1}\left(X^{\prime}\right) \rightarrow \pi_{1}(Y)$, induced by the inclusion $X^{\prime} \subset Y$, is an epimorphism.

Example 4.3. The manifold $Y=S^{k} \times \mathbb{R} P^{2}, k \geqslant 2$, is exceptional since there exists a submanifold $X=S^{k} \times \mathbb{R} P^{1} \subset S^{k} \times \mathbb{R} P^{2}$ with $\pi_{1}(X)=\mathbb{Z}$. On the other hand, the manifold $S^{k} \times \mathbb{R} P^{4}, k \geqslant 2$, is non-exceptional.

In order to verify this, suppose to the contrary. Then there exists a submanifold $X \subset$ $S^{k} \times \mathbb{R} P_{k}^{4}$ such that $\pi_{1}(X)=\underset{i=1}{*} \mathbb{Z}^{+}$. Every epimorphism $\underset{i=1}{*} \mathbb{Z}^{+} \rightarrow \mathbb{Z}_{2}^{+}$factors through the $\operatorname{map} \underset{i=1}{*} \mathbb{Z}^{+} \rightarrow \mathbb{Z}^{+} \rightarrow \mathbb{Z}_{2}^{+}$. Let

$$
p: S^{k} \times \mathbb{R} P^{4} \rightarrow \mathbb{R} P^{N}, \quad N \gg k+4
$$


be the map which induces an isomorphism of fundamental groups, such that $p^{-1}\left(\mathbb{R} P^{N}\right)=$ $X$. Consider $q=\left.p\right|_{X}: X \rightarrow \mathbb{R} P^{N-1}$. The map $q$ factors through the map $q^{\prime}: X \rightarrow$ $S^{1} \subset \mathbb{R} P^{N-1}$. Therefore, $p$ can be chosen so that the preimage $p^{-1}\left(\mathbb{R} P^{N-3}\right)$ is empty. On the other hand, the cocycle $p^{*}\left(t^{3}\right) \in H^{3}\left(S^{k} \times \mathbb{R} P^{4} ; \mathbb{Z}_{2}\right)$, where $t \in H^{3}\left(\mathbb{R} P^{N} ; \mathbb{Z}_{2}\right)$ is a generator, is not cohomologous to 0 . Therefore the manifold $S^{k} \times \mathbb{R} P^{4}$ is non-exceptional.

Proposition 4.4. For every finite group $G$, equipped with the orientation character $\omega$, and for every $n \geqslant 5$, there exists a closed exceptional manifold $Y$ of dimension $\operatorname{dim} Y=$ $n+1$, such that $\pi_{1}(Y) \cong G$ and $\omega=\omega_{1}(Y) \in H^{1}\left(G ; \mathbb{Z}_{2}\right)$.

Proof. Consider the epimorphism $r: \underset{i=1}{*} \mathbb{Z} \rightarrow G$ of the free group on $k$ generators onto the group $G$, and let us construct a manifold $X^{\prime}$ as the connected sum of $k$ copies of the pair $\left(S^{n-1} \times S^{1}, S^{n-1} \tilde{\times} S^{1}\right)$ in accordance with the orientation character on the group $G^{-}$. Consider the 1-fibration $\eta \rightarrow X^{\prime}$ which corresponds to the subgroup $r^{-1}(\pi) \subset \underset{i=1}{*} \mathbb{Z}$ of index 2. A tubular neighbourhood $\partial \eta$ is then the boundary of some manifold $W$, $\partial W=\partial \eta$, and the homomorphism $\pi_{1}(W) \rightarrow \pi$ agrees with the given one on $\partial \eta$.

We perform a surgery on the manifold $W$ to obtain $W^{\prime}$, by means of interior surgeries on handles of index 2 , and we kill the kernel of the map $\pi_{1}(W) \rightarrow \pi$. We have that $\partial W=$ $\partial W^{\prime}$ and we get a well-defined manifold $Y=\eta \cup_{\partial \eta} W^{\prime}$. By construction, $\pi_{1}(Y) \cong G$. Moreover, the submanifold $X \subset Y$ is the result of a surgery on the submanifold $X^{\prime}$ inside $Y$, of handles of index 2 , killing $\operatorname{Ker}\left[\pi_{1}\left(X^{\prime}\right) \rightarrow \pi_{1}(Y)\right]$. The pair $(Y, X)$ is admissible and the manifold $Y$ is exceptional.

Proof of Theorem 2.10. Suppose that the obstruction to surgery of a simple homotopy equivalence $f: M \rightarrow Y$ equals $u(f) \in \mathrm{LN}_{n}(\pi \rightarrow G)$. By construction, $u(f)$ lies in the image of the group $\operatorname{LS}_{n}(Y, X) \rightarrow \mathrm{LN}_{n}(\pi \rightarrow G)$, where $X^{\prime} \subset Y$ is a submanifold with a free fundamental group.

Passing to the normal map $\left.f\right|_{N}: N \rightarrow X$ we observe that the obstruction to surgery of this map in the group $L_{n}\left(G^{-}\right)$lies in the image of the subgroup $L_{n}\left(\underset{i=1}{*} \underset{i}{*} \mathbb{Z}^{ \pm}\right) \rightarrow L_{n}\left(G^{-}\right)$. Finally, from the Mayer-Vietoris sequence (cf. $[3]$ ) we conclude that the group $L_{n}\left(\underset{i=1}{*} \mathbb{Z}^{ \pm}\right)$ is generated by the image of $L_{n}\left(\underset{k}{\mathbb{Z}^{ \pm}}\right) \rightarrow L_{n}\left(\underset{i=1}{\stackrel{*}{*} \mathbb{Z}^{ \pm}}\right)$under the homomorphisms induced by the regular embeddings $\mathbb{Z}^{ \pm} \subset \underset{i=1}{*} \mathbb{Z}^{ \pm}$.

Acknowledgements. P.M.A. was supported by the Russian Foundation for Fundamental Research grant no. 99-01-0009, A.C. by the GNSAGA of the CNR (National Research Council) of Italy, by the Ministero dell' Università e della Ricerca Scientifica e Tecnologica of Italy within the project Geometria Reale e Complessa, and by a research grant of University of Modena, and D.R. by the Ministry for Science and Technology of the Republic of Slovenia grants no. J1-0885-0101-98 and SLO-US 0020. We acknowledge A. A. Ranicki and the referee for many valuable comments and suggestions. 


\section{References}

1. P. M. Akhmetiev and Yu. V. Muranov, Obstructions to the splitting of manifolds with infinite fundamental group, Mat. Zametki 60 (1996), 163-175 (in Russian). (English transl. in Math. Notes 60 (1996), 121-129.)

2. W. Browder and G. R. Livesay, Fixed point free involutions on homotopy spheres, Bull. Am. Math. Soc. 73 (1976), 242-245.

3. S. E. Cappell, Mayer-Vietoris sequences in Hermitian K-theory, Algebraic K-theory, III: Hermitian K-theory and geometric applications, in Proc. Conf. Battelle Memorial Inst., Seattle, WA, 1972, pp. 478-512 (Springer, Berlin, 1972).

4. S. E. Cappell, Groups of singular Hermitian forms, in Proc. Conf. Battelle Memorial Inst., Seattle, WA, 1972, pp. 513-525 (Springer, Berlin, 1972).

5. S. E. Cappell and J. L. Shaneson, A counterexample on the oozing problem for closed manifolds, in Algebraic topology (Aarhus, 1978), in Proc. Symp. Univ. Aarhus, Aarhus, 1978, Lecture Notes in Mathematics, vol. 763, pp. 627-634 (Springer, Berlin, 1979).

6. I. Hambleton, R. J. Milgram, L. Taylor and B. Williams, Surgery with finite fundamental group, Proc. Lond. Math. Soc. 56 (1988), 349-379.

7. A. F. Kharshiladze, Hermitian $K$-theory and quadratic extensions, Trudy Moskov. Mat. Obšč. 41 (1980), 3-36 (in Russian).

8. A. F. Kharshiladze, Surgery on manifolds with finite fundamental groups, Usp. Mat. Nauk 42(4) (1987), 55-85 (in Russian).

9. S. L. de Medrano, Involutions on manifolds (Springer, Berlin, 1971).

10. Yu. V. Muranov and D. Repovš, Groups of obstructions to surgery and splitting for a manifold pair, Mat. Sbornik 188(3) (1997), 127-142 (in Russian). (English transl. Math. Sbornik 188(3) (1997), 449-463.)

11. A. A. Ranicki, Exact sequences in the algebraic theory of surgery, Mathematical Notes, vol. 26 (Princeton University Press, 1981).

12. A. A. Ranicki, Algebraic L-theory and topological manifolds (Cambridge University Press, 1992).

13. J. L. Shaneson, Wall's surgery obstruction groups for $G \times \mathbb{Z}$, Ann. Math. (2) 90 (1969), 296-334.

14. C. T. C. Wall, Surgery on compact manifolds, London Mathematical Society Monographs, vol. 1 (Academic Press, New York, 1970). 